\title{
Vaginal rejuvenation: current perspectives
}

This article was published in the following Dove Press journal:

International Journal of Women's Health

21 July 2017

Number of times this article has been viewed

\author{
Giussy Barbara' \\ Federica Facchin ${ }^{2}$ \\ Laura Buggio 3 \\ Daniela Alberico ${ }^{3}$ \\ Maria Pina Frattaruolo 3 \\ Alessandra Kustermann' \\ 'Department of Obstetrics and \\ Gynecology, Service for Sexual \\ and Domestic Violence (SVSeD), \\ Fondazione IRCCS Ca' Granda, \\ Ospedale Maggiore Policlinico, \\ ${ }^{2}$ Faculty of Psychology, Catholic \\ University of Milan, ${ }^{3}$ Department \\ of Obstetrics and Gynecology, \\ Fondazione IRCCS Ca' Granda, \\ Ospedale Maggiore Policlinico, \\ University of Milan, Milan, Italy
}

Correspondence: Giussy Barbara Department of Obstetrics and Gynecology, Service for Sexual and Domestic Violence (SVSeD), Fondazione IRCCS Ca' Granda, Ospedale Maggiore Policlinico, Via della Commenda I2, Milan MI 20I22, Italy

Tel +390255032359

Email giussy.barbara@gmail.com
Abstract: Female genital cosmetic surgery includes several procedures aimed at reaching better female genital appearance and/or improved sexual functioning. Among these procedures, vaginal rejuvenation is considered as one of the most controversial genital cosmetic surgical interventions and involves a range of surgical procedures performed by gynecologists or plastic surgeons to decrease the average diameter of the vagina, mainly for sexual reasons. In this narrative review, vaginal rejuvenation outcomes are examined in order to clarify the current scenario of the different vaginal rejuvenation techniques, as well as their effectiveness and associated complications. Psychological and ethical issues linked to these procedures are also addressed.

Keywords: vaginal rejuvenation, female genital cosmetic surgery, vaginoplasty

\section{Introduction}

Female genital cosmetic surgery (FGCS) includes several surgical interventions (such as vaginal rejuvenation, labiaplasty, vulvar liposculpturing, re-virgination with hymenoplasty, and G-spot amplification) aimed at reaching better female genital appearance and/or improved sexual functioning. ${ }^{1}$ In recent years, FGCS procedures especially vaginal rejuvenation - have gained increasing popularity in Western countries, not only among health care providers but also among women. In a US survey, the American Society of Plastic Surgeons reported a 30\% increase in the rate of vaginal rejuvenation procedures (from 793 in 2005 to 1,030 in 2006). ${ }^{2}$ All these interventions are performed mainly for esthetic reasons or, in some cases, to increase sexual functioning, although with no clear medical indication. In general, vaginal rejuvenation is often proposed to women who feel a sensation of wide vagina due to vaginal delivery or aging, in order to enhance the vaginal tone and thus sexual friction.

The increasing popularity of FGCS has elicited academic attention worldwide. In 2007, the American College of Obstetricians and Gynecologists (ACOG) published a critical position paper on FGCS, pointing out that vaginal rejuvenation or other FGCS procedures are not accepted surgical practices, as there is paucity of data supporting their safety and efficacy, despite potential complications, such as infection, dyspareunia, scarring, and altered sensation. ${ }^{3}$

Similarly, in a 2013 ethical opinion paper on FGCS, the Royal College of Obstetricians and Gynaecologists (RCOG) underlined that presenting FGCS procedures as an unproblematic lifestyle choice is inappropriate because it misinforms women about the actual efficacy of these procedures. ${ }^{4}$ Moreover, the Royal Australian and New Zealand College of Obstetricians and Gynaecologists (RANZCOG) and the Society of Obstetricians and Gynecologists of Canada (SOGC) took a public stand against FGCS. ${ }^{5,6}$

In particular, vaginal rejuvenation is considered as one of the most controversial FGCS interventions. Vaginal rejuvenation involves a range of procedures performed 
by gynecologists or plastic surgeons to decrease the average diameter of the vagina, mainly for sexual reasons. These procedures are referred to as vaginal rejuvenation, vaginal restoration/rogation, or vaginoplasty. These multiple terms reflect the problematic commercial/marketing interests potentially associated with this surgery, to the point that several medical organizations called for an urgent need for a standardized nomenclature, with the elimination of all non-medical terms.

Against this background, we performed a narrative review of articles reporting vaginal rejuvenation outcomes to clarify the current scenario of this surgery (ie, types of techniques, effectiveness, associated complications), as well as to address psychological and ethical issues. An electronic database search (PubMed) was conducted to identify Englishlanguage journal articles published between January 2000 and September 2016 on vaginal rejuvenation procedures (excluding other types of FGCS). Appropriate search terms were selected by reviewing titles, keywords, and abstracts of a sample of studies. "Female genital cosmetic surgery", "vaginal rejuvenation", "vaginoplasty", "genital aesthetics", "female genital plastic surgery", "vaginal tightening", and "perineum rejuvenation" were used as research terms. Moreover, we further checked the reference lists of all the retrieved articles to identify additional studies. No attempt was made to find unpublished studies. In this narrative review, the best quality evidence was selected with preference given to the most recent articles and reviews. Since only published data were considered, the current research was exempt from the institutional review board approval.

\section{Toward a standardization of female genital esthetics and sexual functioning}

There is evidence that the increasing request of genital cosmetic surgery derives from women's desire for a standardized, pre-pubic genital appearance, namely "Barbie doll look", in which the labia minora are narrow and not visible and the vagina opening appears very tight. ${ }^{7-11}$ This specific minimalistic ideal of female genitalia is culturally dependent and affected by the development of Internet pornography, the popularity of total pubic hair removal, and the standardized representation of female genitalia in magazines and popular culture, despite the wide range of anatomic normality of female external genitalia. ${ }^{7,11-13}$

Following this new ideal of the "perfect vagina", some authors have proposed a novel concept of "genital beautification", which involves a combination of different
FGCS techniques: labia majora augmentation, labia minora reduction, labial brightening by laser, mons pubic reduction, and vaginal tightening. ${ }^{14}$ According to McDougall, ${ }^{9}$ this new female vulvar beauty ideal is like a "clean slit", such that after cosmetic surgery female genitalia appears small, smoothed-tight, and symmetrical, with little color and details, and nothing protruding below the labia majora. Following this conceptualization, the perfect vulvar ideal seems to be what just ends in a perfect point of nothingness. ${ }^{9}$

Internet plays a major role in the pathologization of genital diversity and in the promotion and normalization of FGCS, as many women who are concerned about the appearance of their genitalia are likely to use the web to search for information. There is evidence that women's perception of normal genitalia can be changed by the exposure to different images of female external genitalia. Moran and Lee ${ }^{15}$ reported that exposure to images of surgically modified vulvas may influence women's perceptions of what is normal and desirable. This may explain why the Internet and in general media exposure to standardized images of female genitalia might exacerbate women's insecurities about their appearance. Recent research has also evaluated men's perceptions regarding the vulvar anatomy, due to its potential influences on women's desire for FGCS. ${ }^{16}$ It was found that most men were sufficiently knowledgeable about the anatomy of female external genitalia and, although younger men showed a preference for minimalized labia, the majority of the sample did not believe that vulvar anatomy may have an adverse impact on sexual desire or pleasure. ${ }^{16}$

Overall, online information on vaginal rejuvenation is often provided by private surgery providers, and therefore, it may offer only a partial perspective on this sensitive issue. For instance, these websites use confounding labels to define different FGCS interventions, with little information on potential complications and absent or little evidence for clinical effectiveness. ${ }^{17,18}$ Moreover, associations between anatomy of genitalia and quality of sexual function are often reported, suggesting that genital cosmetic surgery necessarily improves female sexual functioning and partner's sexual satisfaction. ${ }^{17,19-21}$

Internet pornography also contributes to the dissemination of a deformed image of human sexuality as a standardized mechanical "performance" that requires perfect functioning from either an anatomical or a physiological point of view. Such an idea is congruent with the overall medicalization of human sexuality, which could be associated with the increasing phenomenon of disease mongering. ${ }^{22,23}$ As accurately stated by Braun, ${ }^{24}$ a critical point to consider is whether new 
anxieties and commercial needs are being created through the promotion of this kind of surgery and the pathologization of individual physiological differences in vulvar anatomy.

\section{Vaginal rejuvenation: surgical options, efficacy, and complications}

From a surgical point of view, vaginal rejuvenation consists of a combination of different surgical interventions performed to decrease the diameter of the vaginal canal and opening, such as excision of redundant vaginal tissues, muscular tightening by imbrication of the median levator muscles, remodeling of the perineal body, and excision of the surplus skin. The classical surgical technique used during vaginal rejuvenation interventions is very similar to traditional anterior or posterior colporrhaphy performed in urogynecological settings to correct vaginal prolapse or pelvic floor defects. Colporrhaphy is often associated with perineoplasty, which involves the removal of a triangle-shaped portion of skin tissue from the perineum above the anus to tighten the vaginal introitus and reconstruct the perineal body.

Pardo et $\mathrm{al}^{25}$ used colporrhaphy to treat abnormal or subjectively wide vaginas on women with secondary sexual dissatisfaction and impaired/nullability to reach orgasm. The 53 women enrolled in the study underwent a site-specific anterior and posterior repair; in all cases, paravaginal stitches were placed during the anterior repair, and the fasciae were dissected by means of a Yag laser. In almost all cases, surgeons also performed a perineoplasty. At the end of the procedures, vaginal diameter was decreased so that only two fingers could be inserted in at least the lower two-thirds of the vagina. Authors reported that 6 months post-surgery, $66 \%$ of women experienced a great improvement in sexual life, $24 \%$ a significant improvement, whereas just $10 \%$ a slight or no improvement at all. Sexual function was not evaluated with a validated sexual questionnaire. Significantly, before surgery, a huge proportion of women declared that, besides their desire of a tighter vagina, they wanted their partner to feel a tighter vagina too. Authors reported no significant intraoperative complications and only two minor postoperative complications (ie, surgical wound dehiscence).

Abedi et $\mathrm{al}^{26}$ assessed sexual functioning after vaginal tightening performed for a sensation of vaginal laxity. The surgical technique used involved the vertical incision of the vagina opening, the exposure of the rectovaginal tissue and the levator ani muscle, the removal of the appropriate amount of tissue according to the relaxation degree, and the suture of the remaining vaginal tissue. After 6 months, sexual function measured with a validated instrument (ie, the Female
Sexual Function Index [FSFI]) was globally improved, but a high level of new dyspareunia and low vaginal lubrication were noted, limiting the beneficial effects of this type of surgery.

A large multicenter cross-sectional study of $\mathrm{FGCS}^{27}$ including 258 women and 341 different genital plastic interventions was conducted in order to assess several outcomes, such as the reasons for considering surgery, surgical outcomes and complications, and patient satisfaction. In this study, 47 women underwent vaginoplasty/perineoplasty procedures with a high rate of satisfaction in terms of enhancement of sexual functioning for both women and partners.

Other non-traditional surgical procedures proposed for vaginal rejuvenation involve the use of laser technology (ie, fractional $\mathrm{CO}_{2}$ laser, erbium YAG laser) to tight the vagina. Laser vaginal rejuvenation is often advertised as a conservative, minimally invasive surgical technique for the enhancement of sexual functioning in women with a sensation of vaginal laxity, with no complications and high efficacy. It has also been proposed to women with dyspareunia or fibrosis of the vaginal mucosae as a result of episiotomies or previous vaginal surgery, as well as to women with altered genital sensations because of vaginal atrophy for hormonal changes due to menopause and aging. ${ }^{28}$ The majority of information on these type of procedures derives from the web, and the scientific literature on this specific topic is very limited.

Laser $\mathrm{CO}_{2}$ is generally used in dermatologic and esthetic surgery for the treatment of several skin and mucosal lesions, such as acne scars or chrono-aging facial defects. Laser energy in the mid-infrared invisible light spectrum stimulates collagen/elastin contraction, neocollagenesis, and neovascularization and thus improves vaginal lubrication by revitalizing and restoring the elasticity and hydration of the female lower genital tract mucosa. Laser treatment sessions usually last 10-20 minutes; sometimes patients report a local warming sensation not requiring the use of anesthetics, and regular activities can be resumed early. ${ }^{29}$

However, some authors have raised concerns about the safety of laser procedures, as tissue damage and remodeling resulting from laser use may potentially increase urogenital pain and dyspareunia; moreover, the distal effect of the laser energy on proximal organs such as rectum, urethra, and bladder is actually unknown. ${ }^{28}$

Salvatore et $\mathrm{al}^{30}$ investigated the effects of fractional $\mathrm{CO}_{2}$ laser on sexual function and satisfaction in 77 postmenopausal women with vulvovaginal atrophy. Quality of life and sexual functioning were evaluated with validated international questionnaires (ie, the Short Form-12 [SF-12] 
and the FSFI) at baseline and after 12 weeks; the intensity of vaginal symptoms, such as vaginal burning, dryness, itching, and dyspareunia, was evaluated using a $10 \mathrm{~mm}$ visual analog scale. At the 12-week follow-up, the authors found a significant improvement in the FSFI total score and domain scores (ie, desire, arousal, lubrication, pain, orgasm, satisfaction). ${ }^{30}$

The effect of fractional $\mathrm{CO}_{2}$ laser on women reporting symptoms of vulvovaginal atrophy (dryness, itching, burning, dyspareunia, discomfort, urinary symptoms) was recently evaluated by Filippini et $\mathrm{al}^{31}$ in 386 postmenopausal women. In menopause, due to the lack of the estrogenic support to the genital mucosa, vaginal rejuvenation often involves a restoration of the vaginal wall connective tissue and the adequate tissue water content, besides a mere anatomical surgical correction to tighten the vaginal diameter. The authors found that fractional micro-ablative $\mathrm{CO}_{2}$ laser was effective in treating the vaginal epithelium and improving uncomfortable symptoms and dyspareunia due to vaginal atrophy. ${ }^{31}$

Vizintin et $\mathrm{al}^{32}$ used the effects of the erbium YAG laser SMOOTH technology to treat vaginal laxity and consequently improve female sexual satisfaction. YAG laser has non-ablative, thermal-only effects and has been used to produce vaginal collagen hyperthermia and remodeling, resulting in improved vaginal tissue elasticity and tightness. The research protocol was based on heating the vaginal wall mucosa up to $\sim 65^{\circ} \mathrm{C}$ and included two treatment sessions with a 4- to 6-week interval. Based on their findings, the authors concluded that SMOOTH-mode erbium laser may be an effective and safe method for treating vaginal laxity.

Another minimally invasive surgical technique proposed for vaginal rejuvenation is the radiofrequency (RF) treatment. ${ }^{33-37}$ Several RF devices, which emit focused electromagnetic waves, are currently available on the market, with a specific focus on vaginal rejuvenation. None of these treatments require the use of anesthetics, and the treatment sessions last 15-30 minutes. Historically, non-ablative RF energy has been used in esthetic medicine for conservative treatment of lax skin of the face and neck and in the delicate tissue of the periorbital area because of the thermal tissue remodeling rather than ablative effects. ${ }^{33-36}$

Millheiser et $\mathrm{al}^{37}$ evaluated safety and tolerability of reverse gradient RF energy $\left(75-90 \mathrm{~J} / \mathrm{cm}^{2}\right)$, delivered through the vaginal mucosae, in 24 women with vaginal laxity after delivery. No adverse events were reported, and no topical anesthetic was required. The majority of women $(87 \%)$ reported a subjective sensation of improved vaginal tightness after 6 months. Women also reported a substantial amelioration of sexual functioning, measured with validated questionnaires. Transcutaneous temperature-controlled radiofrequency (TTCRF) was used on vulvovaginal tissue for orgasmic dysfunction, and authors reported that patients also noted significant vaginal tightening effects. ${ }^{37}$

In another study by Sekiguchi et al, ${ }^{38} 30$ premenopausal women were treated with a single session of RF therapy at $90 \mathrm{~J} / \mathrm{cm}^{2}$. Authors reported a significant improvement in sexual functioning and a decreased distress related to sexual activity at the 6-month follow-up. Women also reported decreased vaginal laxity within the first months after the RF treatment. ${ }^{38}$

Ostrzenski ${ }^{39}$ proposed another method for treating vaginal laxity, ie, vaginal rugation rejuvenation (restoration). In his article, the author first defined smooth vagina as characterized by absent or decreased vaginal rugation, which can lead to decreased sensation of penile friction during coitus. Decreased vaginal rugae seem to be related to advancing age and low estrogenic levels. The surgical procedure involved the use of $\mathrm{CO}_{2}$ laser in the continuous mode of 8-10 W with defocus. The depth of vaginal columnar rugae vaporization was between 2 and $5 \mathrm{~mm}$ width, and vaporization should stop at the level of endopelvic fascia. Amelioration of sexual function was observed in the 10 women participants, with no reported complications.

Vulvovaginal lipofilling and injection of hyaluronic acid and combined platelet-rich plasma was proposed as a novel procedure for vulvovaginal rejuvenation. ${ }^{40}$ The surgical technique involves a vaginoplasty by lipofilling of the posterior vaginal wall, with an injection of hyaluronic acid and combined platelet-rich plasma subcutaneously in the perineum area. In a case report study ${ }^{40}$ on this particular procedure, this technique seems to be safe and easy to perform, although more research is needed to provide firm evidence of its effectiveness and safety.

Some authors recently introduced a novel procedure for vaginal rejuvenation, ie, by using Gore-Mycromesh, which is a biocompatible material composed of expanded poly-tetrafluoroethylene (ePTEE), commonly used in plastic and reconstructive surgery for inguinal or femoral hernias or for genital prolapse. ${ }^{41}$ The study was conducted on 50 women who underwent surgery for vaginal laxity to improve their sexual functioning, assessed with the FSFI. The surgical technique consisted of an insertion of a GoreMycromesh $\left(2 \times 4 \mathrm{~cm}^{2}\right.$ in size) under the submucosae of the posterior vaginal wall under local anesthesia. The authors found a substantial improvement in sexual function 1 year after surgery, especially in the FSFI satisfaction domain. 
No significant complications, such as infection or mesh extrusion, were reported. ${ }^{41}$ The same authors also proposed a functional vaginal rejuvenation with elastic silicone threads in 180 women with a feeling of wide vagina. ${ }^{42}$ This novel surgical technique requires the insertion of silicone threads under the vaginal submucosae, following two incisions at 3 and 9 o'clock for the inlet and outlet of the threads. In this kind of intervention, it is crucial to avoid injuring the urethra (anterior to vagina) and the rectum (posterior to vagina). Overall, the authors reported that after 12 months, the FSFI total score was significantly improved, particularly regarding the FSFI orgasm domain; moreover, $92.8 \%$ of the women were satisfied with outcomes with regard to feeling of correction of vaginal width. The most common complications reported were implant exposure (5\% of women), capsule contracture $(3.9 \%)$, and infection $(1.7 \%){ }^{42}$

\section{Discussion and conclusion}

Vaginal rejuvenation is described as a safe and effective surgical procedure for the enhancement of sexual functioning in women with an acquired sensation of vaginal laxity and consequent sexual dissatisfaction. Besides the more traditional surgical techniques (anterior and posterior vaginoplasty and colporrhaphy), there has been a recent increase in the proposal of innovative, non-invasive procedures $\left(\mathrm{CO}_{2}\right.$ laser, RF) claimed to be effective and associated with no or few post-surgical complications. However, the primary concern is that there is currently poor scientific evidence of the effectiveness of these procedures.

The different techniques proposed for vaginal rejuvenation have been studied in small trials assessing surgical outcomes, patient satisfaction, and postoperative complications. There is an urgent need for more robust data (possibly deriving from multicenter randomized controlled studies) before considering these practices as safe and effective. ${ }^{43,44}$ The primary source of information describing the various vaginal rejuvenation procedures is represented by several different websites of private surgeons or centers of cosmetic and esthetic surgery. This raises concerns about the possible existence of financial/personal conflicts of interest, not explicitly declared, that could affect the transparency and the accuracy of the reported beneficial outcomes.

The debate around the need for robust evidence of the effectiveness and safety of FGCS, also supported by several academic and scientific societies, ${ }^{3-6}$ is particularly important as regards vaginal rejuvenation procedures. In fact, despite some genital surgical interventions (for instance, labiaplasty) are aimed at modifying the morphology of external genitalia mainly for esthetic reasons, vaginal rejuvenation procedures are primarily performed for a functional reason, that is enhancing the sexual satisfaction of women and their partners. In these cases, the most important criterion for choosing such a procedure is the observed effectiveness and safety, which should be adequately defined.

Vaginal rejuvenation procedures have drawn criticism for various reasons, but particularly because they cannot be properly classified as esthetic surgical interventions. Whereas accepted cosmetic/plastic surgery interventions (such as breast augmentation or reduction, nose reshaping, liposuction, facelift) are specifically performed to improve appearance and enhance self-confidence, this does not properly seem to be the case of vaginal rejuvenation procedures.

Moreover, some authors have raised concerns that FGCS would be somehow comparable to female genital mutilation (FGM) - which is the cultural practice of removing part or all of the external genitalia of women - not only in terms of anatomical alterations but also in terms of direct and indirect unethical societal pressures that lead women to undergo genital cosmetic surgery. ${ }^{45}$

Surgeons performing vaginal rejuvenation interventions should exhaustively inform their patients that there is little evidence supporting this kind of surgery to increase sexual satisfaction, such that they are operating without a robust evidence base. ${ }^{3,4,6}$ Moreover, they should inform women that these procedures, besides a lack of documented effectiveness, are associated with potential post-surgical complications. ${ }^{3}$ However, it is conceivable that in case of significant anatomical alteration (such as genital prolapse or as a consequence of a complicated vaginal delivery), surgical interventions performed to tighten the vagina could increase sexual functioning in women and their partners, as several studies on surgical correction of female genital prolapse showed an improvement in patients' sexual functioning. ${ }^{46-48}$

One of the main issues while performing vaginal rejuvenation for sexual reasons is that the precise nature of sexual dysfunctions should be preliminary ruled out and extensively discussed with women to avoid unrealistic expectations, since in many cases, surgery is not appropriate and may not lead to a definitive solution. Another problem is that in several studies, the notion of vaginal laxity, prerequisite for vaginal rejuvenation, is vague and subjective. Vaginal laxity can derive from a relaxation of the vaginal wall, from anterior or posterior compartment prolapse, or from a distension of the fibers of the puborectalis muscle. All these different anatomical reasons for vaginal laxity require different kinds 
of surgical repair, and the correct pre-operatory definition of the nature of the defect is essential in choosing the appropriate surgical procedure to tighten the vagina. ${ }^{44}$

In conclusion, we deem it necessary to underline that the contemporary idea of sexuality as a mechanical performance, the diffusion of which has been facilitated by the Internet (especially Internet pornography), including the social media, is distorted, reductionist, and therefore dangerous. Anatomic perfection does not represent the necessary condition for satisfying and healthy sexuality, which depends on the interaction of multiple factors, including cultural, relational, and psychological dimensions. For instance, there is evidence of the association between body dysmorphic disorder and the request for cosmetic surgery; ${ }^{49}$ this means that some individuals are not able to acknowledge the psychic origin of their emotional sufferance, which they consider as caused by actual bodily flaws to the point of seeking multiple interventions of cosmetic surgery. For this reason, we strongly recommend that psychological and sexological counseling is proposed to all women who seek FGCS; on one hand, it may help rule out the possible presence of undiagnosed and thus untreated psychiatric symptoms; on the other hand, it may help some women identify that their problems, including sexual dysfunctions, may have other origins, such as the quality of the couple's relationship. How this multidisciplinary ethical approach to FGCS could be compatible with the interests of the overall cosmetic surgery business remains a major concern.

\section{Disclosure}

The authors report no conflicts of interest in this work.

\section{References}

1. Iglesia CB. Cosmetic gynecology and the elusive quest for the "perfect vagina". Obstet Gynecol. 2012;119(6):1083-1084.

2. Lowenstein L, Salonia A, Shechter A, Porst H, Burri A, Reisman Y. Physicians' attitude toward female genital plastic surgery: a multinational survey. J Sex Med. 2014;11(1):33-39.

3. Committee on Gynecologic Practice. American College of Obstetricians and Gynecologists. ACOG Committee Opinion No 378: vaginal "rejuvenation" and cosmetic vaginal procedures. Obstet Gynecol. 2007; 110(3):737-738.

4. Royal College of Obstetricians and Gynaecologist. Ethical considerations in relation to female genital cosmetic surgery (FGCS). RCOG Ethics Committee, 2013. Available from: https://www.rcog.org.uk/globalassets/ documents/guidelines/ethics-issues-and-resources/rcog-fgcs-ethicalopinion-paper.pdf. Accessed June 26, 2017.

5. The Royal Australian and New Zealand College of Obstetricians and Gynaecologists. RANZCOG College Statement: C-Gyn 24. Vaginal "Rejuvenation" and Cosmetic Vaginal Procedures; 2008. Available from: https://www.ranzcog.edu.au/RANZCOG_ SITE/media/DOCMAN-ARCHIVE/Vaginal $\% 20$ rejuvenation $\% 20$ and $\% 20$ cosmetic\%20vaginal $\% 20$ procedures $\% 20(C-G y n \% 2024) \% 20$ Amended\%20July\%202016.pdf. Accessed June 26, 2017.
6. Committee Clinical Practice Gynaecology, Ethics Committee, and Executive Council of the Society of Obstetricians and Gynaecologists of Canada. Female genital cosmetic surgery. J Obstet Gynaecol Can. 2013; 35(12):e1-e5.

7. Schick VR, Rima BN, Calabrese SK. Evulvalution: the portrayal of women's external genitalia and physique across time and the current Barbie doll ideals. J Sex Res. 2010;48(1):74-81.

8. Braun V, Kitzinger C. The perfectible vagina: size matters. Cult Health Sex. 2001;3(3):263-277.

9. McDougall LJ. Towards a clean slit: how medicine and notions of normality are shaping female genital aesthetics. Cul Health Sex. 2013; 15(7):774-787.

10. Davis SW. Loose lips sink ships. Fem Stud. 2002;28(1):7-35.

11. Tiefer L. Female genital cosmetic surgery: freakish or inevitable? Analysis from medical marketing, bioethics, and feminist theory. Feminism Psychol. 2008;18(4):466-479.

12. Bramwell R. Invisible labia: the representation of female external genitals in women's magazines. Sex Relat Ther. 2002;17(2):187-190.

13. Braun V, Wilkinson S. Socio-cultural representations of the vagina. J Reprod Infant Psychol. 2001;19(1):17-32.

14. Cihantimur B, Herold C. Genital beautification: a concept that offers more than reduction of the labia minora. Aesthetic Plast Surg. 2013; 37(11):1128-1133.

15. Moran C, Lee C. What's normal? Influencing women's perceptions of normal genitalia: an experiment involving exposure to modified and nonmodified images. BJOG. 2014;121(6):761-766.

16. Mazloomdoost D, Crisp CC, Westermann LB, Benbouajili JM, Kleeman SD, Pauls RN. Survey of male perceptions regarding the vulva. Am J Obstet Gynecol. 2015;213(5):731.e1-731.e9.

17. Liao LM, Taghinejadi N, Creighton SM. An analysis of the content and clinical implications of online advertisements for female genital cosmetic surgery. BMJ Open. 2012;2(6):e001908.

18. Mowat H, McDonald K, Dobson AS, Fisher J, Kirkman M. The contribution of online content to the promotion and normalisation of female genital cosmetic surgery: a systematic review of the literature. $B M C$ Womens Health. 2015;15:110.

19. Zwier S. "What motivates Her": motivations for considering labial reduction surgery as recounted on Women's online communities and Surgeons' websites. Sex Med. 2014;2(1):16-23.

20. Moran C, Lee C. Selling genital cosmetic surgery to healthy women: a multimodal discourse analysis of Australian surgery websites. Crit Discourse Stud. 2013;10(4):373-391.

21. Ashong AC, Batta HE. Sensationalising the female pudenda: an examination of public communication of aesthetic genital surgery. Glob $J$ Health Sci. 2012;5(2):153.

22. Tiefer L. Activism on the medicalization of sex and female genital cosmetic surgery by the New View Campaign in the United States. Reprod Health Matters. 2010;18(35):56-63.

23. Barbara G, Facchin F, Meschia M, Vercellini P. Female genital cosmetic surgery: beyond a mechanistic view of sexual satisfaction. Acta Obstet Gynecol Scand. 2015;94(9):1029.

24. Braun V. Female genital cosmetic surgery: a critical review of current knowledge and contemporary debates. $J$ Womens Health (Larchmt). 2010;19(7):1393-1407.

25. Pardo JS, Sola VD, Ricci PA, Guiloff EF, Freundlich OK. Colpoperineoplasty in women with a sensation of a wide vagina. Acta Obstet Gynecol Scand. 2006;85(9):1125-1127.

26. Abedi P, Jamali S, Tadayon M, Parhizkar S, Mogharab F. Effectiveness of selective vaginal tightening on sexual function among reproductive aged women in Iran with vaginal laxity: a quasi-experimental study. J Obstet Gynaecol Res. 2014;40(2):526-531.

27. Goodman MP, Placik OJ, Benson RH 3rd, et al. A large multicenter outcome study of female genital plastic surgery. J Sex Med.2010;7(4 pt 1): $1565-1577$.

28. Singh A, Swift S, Khullar V, Digesu GA. Laser vaginal rejuvenation: not ready for prime time. Int Urogynecol J. 2015;26(2):163-164.

29. Karcher C, Sadick N. Vaginal rejuvenation using energy-based devices. Int $J$ Womens Dermatology. 2016;2:85-88. 
30. Salvatore S, Nappi RE, Parma M, et al. Sexual function after fractional microablative $\mathrm{CO}(2)$ laser in women with vulvovaginal atrophy. Climateric. 2015;18(2):219-225.

31. Filippini M, Del Duca E, Negosanti F, et al. Fractional CO2 laser: from skin rejuvenation to vulvo-vaginal reshaping. Photomed Laser Surg. 2017;35(3):171-175.

32. Vizintin Z, Lukac M, Kazic M, Tettamanti M. Erbium laser in gynecology. Climacteric. 2015;18(suppl 1):4-8.

33. Alinsod RM. Transcutaneous temperature controlled radiofrequency for orgasmic dysfunction. Lasers Surg Med. 2016;48(7):641-645.

34. Magon N, Alinsod R. ThermiVa: the revolutionary technology for vulvovaginal rejuvenation and noninvasive management of female SUI. J Obstet Gynecol India. 2016;66(4):300-302.

35. Weiss RA, Weiss MA, Munavalli G, Beasley KL. Monopolar radiofrequency facial tightening: a retrospective analysis of efficacy and safety in over 600 treatments. J Drugs Dermatol. 2006;5(8):707-712.

36. Fitzpatrick R, Geronemus R, Goldberg D, Kaminer M, Kilmer S, Ruiz-Esparza J. Multicenter study of noninvasive radiofrequency for periorbital tissue tightening. Lasers Surg Med. 2003;33(4):232-242.

37. Millheiser LS, Pauls RN, Herbst SJ, Chen BH. Radiofrequency treatment of vaginal laxity after vaginal delivery: nonsurgical vaginal tightening. J Sex Med. 2010;7(9):3088-3095.

38. Sekiguchi Y, Utsugisawa Y, Azekosi Y, et al. Laxity of the vaginal introitus after childbirth: nonsurgical outpatient procedure for vaginal tissue restoration and improved sexual satisfaction using low-energy radiofrequency thermal therapy. $J$ Womens Health. 2013;22(9): 775-781.

39. Ostrzenski A. Vaginal rugation rejuvenation (restoration): a new surgical technique for an acquired sensation of wide/smooth vagina. Gynecol Obstet Invest. 2012;73(1):48-52.
40. Aguilar P, Hersant B, SidAhmed-Mexi M, Bosc R, Vidal L, Menigaud JP. Novel technique of vulvo-vaginal rejuvenation by lipofilling and injection of combined platelet-rich-plasma and hyaluronic acid: a case-report. Springerplus. 2016;5(1):1184.

41. Park TH, Whang KW. Vaginal rejuvenation with Gore-Mycromesh. Aesthetic Plast Surg. 2015;39(4):491-494.

42. Park TH, Park HJ, Whang KW. Functional vaginal rejuvenation with elastic silicone threads: a 4-year experience with 180 patients. J Plast Surg Hand Surg. 2015;49(1):36-39.

43. Digesu A. Laser vaginal rejuvenation: not ready for prime time-response to comments by Maggiore et al. Int Urogynaecol J. 2015;26(5):785.

44. Barbara G, Facchin F, Meschia M, Vercellini P. Reply "vaginal rejuvenation": comparative effectiveness studies are needed. Acta Obstet Gynecol Scand. 2015;94:1396-1397.

45. Kelly B, Foster C. Should female genital cosmetic surgery and genital piercing be regarded ethically and legally as female genital mutilation? BJOG. 2012;119:389-392.

46. Rogers RG. Sexual function in women with pelvic floor disorders. Can Urol Assoc J. 2013;7(9-10 suppl 4):S199-S201.

47. Glavind K, Larsen T, Lindquist AS. Sexual function in women before and after surgery for pelvic organ prolapse. Acta Obstet Gynecol Scand. 2015;94(1):80-85

48. Kammerer-Doak D. Assessment of sexual function in women with pelvic floor dysfunction. Int Urogynecol J Pelvic Floor Dysfunct. 2009; 20(suppl 1):S45-S50.

49. Barbara G, Facchin F, Meschia M, Vercellini P. "The first cut is the deepest": a psychological, sexological and gynecological perspective on female genital cosmetic surgery. Acta Obstet Gynecol Scand. 2015;94(9):915-920.
International Journal of Women's Health

\section{Publish your work in this journal}

The International Journal of Women's Health is an international, peerreviewed open-access journal publishing original research, reports, editorials, reviews and commentaries on all aspects of women's healthcare including gynecology, obstetrics, and breast cancer. The manuscript management system is completely online and includes

\section{Dovepress}

a very quick and fair peer-review system, which is all easy to use. Visit http://www.dovepress.com/testimonials.php to read real quotes from published authors. 